\title{
Velocity Variations of the roAp Star $\gamma$ Equulei: Probing a puzzling pulsator
}

\author{
Jaymie M. Matthews and Sandra Scott \\ Department of Geophysics \& Astronomy \\ University of British Columbia, Vancouver, V6T 1Z4, Canada
}

\begin{abstract}
We present a pulsational RV curve for $\gamma$ Equ whose amplitude is about $200 \mathrm{~m} / \mathrm{s}$ and period is roughly 11 minutes. These data, combined with rapid photometry and line-profile variations, are being used to identify the nonradial mode(s) and study mode growth/decay in this unusual star.
\end{abstract}

\section{The long and short of $\gamma$ Equ}

The peculiar magnetic CP2 star HD 201601 ( $\gamma$ Equulei) represents two extremes of stellar variability. On the one hand, based on its very slow magnetic variations (between about +500 and $-1000 \mathrm{G}$ ), it is believed to have a rotation period longer than 75 years! At the same time, this object is also a rapidly oscillating Ap (roAp) star, with high-overtone nonradial pulsations of periods near 12 minutes.

The rapid radial velocity (RV) variations associated with roAp pulsations are challenging to measure - with amplitudes of less than a few $\times 100 \mathrm{~m} / \mathrm{s}$. Among the more than two dozen roAp stars known, there have been only two firm detections of RV oscillations: HR 1217 (Matthews et al. 1988) and $\gamma$ Equ (Libbrecht 1988). The latter was based only on low-level peaks in a Fourier periodogram of an RV time series.

Because RV-to-light amplitude ratios and phase lags are a potential mode diagnostic, we have undertaken a major spectroscopic analysis of $\gamma$ Equ.

\section{Observations and analysis}

In collaboration with W.H. Wehlau (UWO), G.A.H. Walker (UBC) and S. Yang (UVic), we obtained over 1620 individual high-resolution spectra $(R \simeq 60,000)$ of $\gamma$ Equ during $19-23$ August 1988, using the CFHT coudé spectrograph and 1872 Reticon detector. Each exposure was about $30 \mathrm{sec}$ long and synchronised with the best estimate of the star's photometric oscillation period, sampling 20 phases per cycle. These exposures were binned in pulsational phase to improve $\mathrm{S} / \mathrm{N}$ and the binned spectra cross-correlated to measure relative velocity shifts. Superposed on each spectrum was a mercury emission line from an arc source at the spectrograph pupil. The apparent shifts in this line were used to gauge instrumental stability and remove drifts. The resulting RV curve for the night of 23 Aug 1988 UT (which shows the largest amplitude) is presented in Fig. 1. 


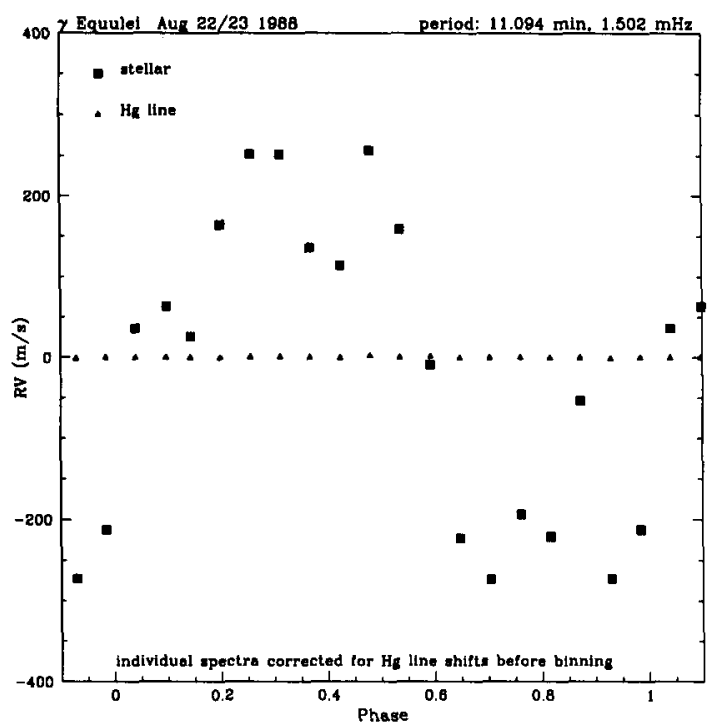

Figure 1. The phased RV curve. Solid symbols are the stellar data; open triangles, the mercury line fiducial to check instrumental stability.

\section{Continuing surprises from $\gamma$ Equ}

The stellar RV curve in Fig. 1 is surprising in two ways: (1) its period, which differs significantly from the dominant photometric period observed on the same night, and (2) its relatively "large" amplitude of about $250 \mathrm{~m} / \mathrm{s}$. The latter is a particular puzzle, given an upper limit of about $0.2 \mathrm{mmag}$ on any light oscillations at the same frequency. Based on RV-to-light amplitude ratios predicted for nonradial pulsators, we would naively expect a photometric amplitude of $2-4 \mathrm{mmag}$. The mystery is compounded by the fact that Libbrecht's (1988) Fourier analysis of his spectral time series yielded an RV amplitude of only 20 $\mathrm{m} / \mathrm{s}$. Weiss \& Schneider (1984) set an upper limit around $50 \mathrm{~m} / \mathrm{s}$ for $\gamma$ Equ.

The data from our entire four-night run confirm the findings of previous observers that the oscillations of $\gamma$ Equ undergo amplitude modulation (or mode evolution) on timescales of a day or less. This is unlikely to be rotational modulation as seen in other roAp stars, if the rotation period of $\gamma$ Equ is indeed as long as decades. The star also shows evidence of profile variability in some lines - the first time this has been seen in an roAp star. We are currently interpreting these data.

\section{References}

Libbrecht, K.A. 1988, ApJ, 330, L51

Matthews, J.M., et al. 1988, ApJ, 324, 1099

Weiss, W.W. \& Schneider, H. 1984, A\&A, 135, 141 